\title{
Hadron spectroscopy from lattice quantum chromodynamics
}

\author{
Sinéad M. Ryan ${ }^{1, \star}$ \\ ${ }^{1}$ School of Mathematics, Trinity College, Dublin 2, Ireland
}

\begin{abstract}
Lattice calculations of hadron spectroscopy are discussed. A brief introduction to numerical simulations of QCD, with a focus on spectroscopy is given. Results for spectroscopy of low-lying, excited and exotic states including hybrids and the $X, Y, Z$ states in charmonium are described. New developments that have allowed for determinations of scattering parameters, including in a coupled-channel analysis are briefly described providing a roadmap for future lattice calculations of excited and exotic states.
\end{abstract}

\section{Introduction}

The study of hadronic energies and strong decay properties is a flourishing field of study. In recent years, hadron spectroscopy has been invigorated by the experimental discovery of unpredicted states in the charmonium and bottomonium systems, some of which are manifestly exotic and cannot be described by quark models. In addition, the unexpected properties of some open-charm mesons, which are narrower and lighter than predicted in quark models has reinvigorated the theoretical study of this sector. We are fortunate that hadron spectroscopy is begin explored by many experiments that can probe these new states with complementary focus and that planned experiments at GlueX and PANDA will bring additional experimental insight and discovery.

In this talk I will discuss lattice hadron spectroscopy with a focus on exotic and hybrid states, in particular in the meson sector where much of the progress and many recent results can be found. In quark models of QCD, the allowed states (the natural spin-parity series) have $P=(-1)^{J}, S=1, C P=$ +1 whereas states with $P=(-1)^{J}$ but $C P=-1$ are forbidden. These so-called exotic states have corresponding $J^{P C}=0^{+-}, 0^{--}, 1^{-+}, 2^{+-}, \ldots$ and while they do not appear in quark models they are allowed by QCD and can be studied on the lattice. Hybrid mesons and baryons are characterised by excited gluonic content, and can have exotic or conventional quantum numbers. When charge conjugation is not a good quantum number (e.g. open charm) there are no exotic states but hybrids are permitted, and can be determined in a lattice calculation, as we will see below.

\section{Lattice QCD}

The formulation of QCD on a four-dimensional hypercubic lattice with a lattice spacing, $a$ and a finite extent, $L$, provides a nonperturbative regulator for the theory and facilitates numerical simulation of QCD observables. This ab initio approach starts from the QCD lagrangian $\mathcal{L}=\bar{\psi}\left(i \gamma_{\mu} D_{\mu}-m\right)-$

^e-mail: ryan@maths.tcd.ie 
$\frac{1}{4} F_{\mu \nu}^{a} F_{a}^{\mu \nu}$. Quark fields $\psi$ are placed on sites of the lattice and carry colour, flavour and Dirac indices, as in the continuum. The lattice gluon fields are described by $S U(3)$ matrices - the links of the hypercube - and derivatives in the continuum theory are replaced with finite differences. Observables, $O$ are then determined from the (euclidean) QCD path integral

$$
\langle O\rangle=\frac{1}{Z} \int \mathcal{D} U_{\mu} \mathcal{D} \bar{\psi} \mathcal{D} \psi O e^{i S_{\mathrm{QCD}}} \stackrel{t \rightarrow i \tau}{\longrightarrow} \frac{1}{Z} \int \mathcal{D} U_{\mu} \mathcal{D} \bar{\psi} \mathcal{D} \psi O e^{-S_{\mathrm{QCD}}},
$$

where the rotation to Euclidean time ensures a non-negative weight that can be used for importance sampling. The quark fields are analytically integrated which introduces the fermion determinant, $M$ in the gauge generation. Practically, calculations proceed by generating an ensemble of gauge field configurations, according to a probability weight given by the gluon action and for a fermion determinant, $M$. Observables, $O$ are evaluated on each gauge field and the ensemble average gives the quantity's value in that simulation with an associated statistical error determined from the standard deviation.

\subsection{Computing correlation functions}

For meson and baryon spectroscopy we consider two-point correlation functions. As an example, consider a local meson operator $\phi(x)=\bar{\psi}_{a}(x) \Gamma \psi_{b}(x)$, where $\Gamma$ is an element of the Dirac algebra with possible displacements and flavour indices $a$ and $b$. A simple two-point function is $C(t)=\left\langle\phi(x) \phi^{\dagger}(0)\right\rangle$. Exploiting euclidean time evolution and inserting a complete set of states yields $C(t)=\sum_{k}|\langle 0|\phi| k\rangle|^{2} e^{-E_{k} t}$. and the ground state energy, $E_{0}$ is determined at large euclidean time $t$ by an exponential fit to the correlator. To access higher-lying energies, a matrix of correlators is constructed from single meson, $\bar{\psi} \Gamma \psi$ and multi-hadron e.g. $\phi=\left(\bar{\psi} \Gamma_{1} \psi\right)\left(\bar{\psi} \Gamma_{2} \psi\right)$, operators and energies are determined by solving a generalised eigenvalue problem $C_{i j}(t) v_{j}^{(n)}=\lambda^{(n)}(t) C_{i j}\left(t_{0}\right) v_{j}^{(n)}$, yielding eigenvalues $\lambda^{(n)}(t) \sim e^{-E_{n} t}\left[1+O\left(e^{-\Delta E t}\right)\right]$ and eigenvectors $v_{j}^{(n)}$ related to the operator overlaps, $Z_{j}^{(n)}=\langle 0|\phi| n\rangle[1]$.

The practicalities of lattice calculations, mean that simulations are frequently performed at heavier-than-physical pion masses and at a single lattice spacing. This is particularly relevant for recent pioneering results on scattering and coupled-channel analyses and a full systematic error budget can be expected as methods mature.

\section{Results}

There has been remarkable progress in lattice spectroscopy in recent years with effort focused both on the low-lying stable states and on excited and exotic hadrons, including resonances. The results discussed here are a snapshot of recent activity - more comprehensive reviews and discussion can be found in Ref. [2]. Much of the focus in lattice studies of exotics and resonances has to date been in the meson sector and the results shown here will reflect that.

\subsection{Stable hadrons below thresholds}

Considering first the spectra of stable states, lying safely below decay thresholds. Many lattice calculations now address all systematic uncertainties by simulating at physical values of the quark masses, with dynamical quarks $\left(N_{f}=2,2+1,2+1+1\right)$ and including continuum extrapolations and in some cases QED effects. Fig. 1 shows a compilation of results of low-lying states ( upper plot, taken from Ref. [3]) and a recent result including QED effects (lower plot, taken from Ref. [4]). In the calculations shown there is a robust error determination and impressive agreement between different lattice calculations. The calculation of baryon electromagnetic splittings includes predictions subsequently verified by experiment. 

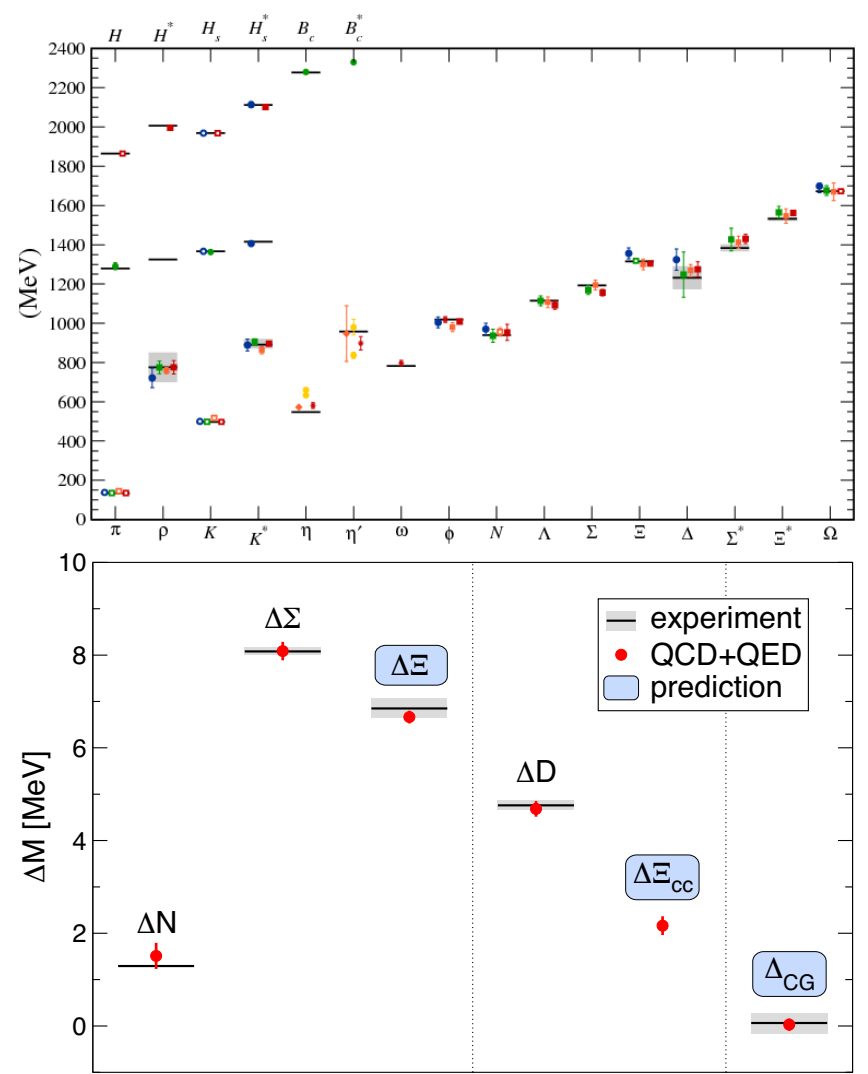

Figure 1. The upper plot shows precision lattice results for a range of low-lying states with all systematic uncertainties under control. The lower plot shows a calculation of electromagnetic splittings in the light baryon sector, with experimental results.

\subsection{Excited and exotic hadrons: as stable states}

The first step in understanding the spectrum of states above decay thresholds - which includes exotic and hybrid states - is to demonstrate that lattice methods for extracting energies and spin identification are working well, yielding precise and robust results for $J \geq 2$ as well as for ground states. The Hadron Spectrum Collaboration has pioneered a suite of methods to do this, enabled by distillation for quark propagation [5]. This approach has been successfully applied to light, heavy-light and heavy hadron spectroscopy [6-14], illustrated in Fig. 2 for the light isoscalar and charmonium spectrum, respectively $[7,10]$. Note that in each case a number of mesons with manifestly exotic quantum numbers are determined - highlighted in the rightmost panel in each plot.

\subsubsection{Hybrids}

The hybrid mesons discussed in the introduction are a focus for experiments including GlueX and PANDA. Such states, with both exotic and conventional quantum numbers can be determined on the lattice and the most extensive study has been carried out by the Hadron Spectrum Collaboration for 


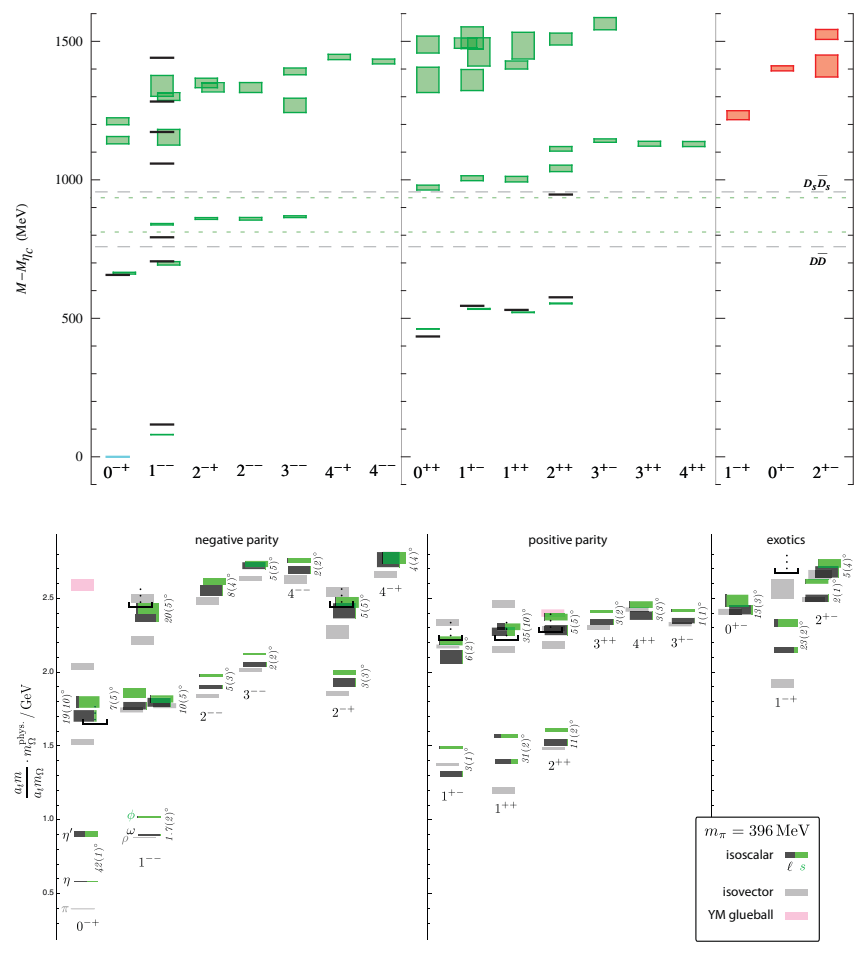

Figure 2. The charmonium (upper plot) and light meson isoscalar (lower plot) spectra determined by the Hadron Spectrum Collaboration [7, 10]. Colored boxes are the lattice results including statistical uncertainty and experimental results, where available, are black lines.

meson and baryon hybrids as part of spectroscopy calculations described above. In a lattice calculation, a state is identified as a candidate hybrid when it has a relatively large overlap onto an operator that is proportional to the field strengh tensor (the commutator of two covariant derivatives on the lattice). Studies with both heavy and light quarks have found the lightest exotic meson in the associated spectrum is a candidate hybrid and is nearly degenerate with three states in the negative partity sector also identified as (non-exotic) hybrids. As a representative result, Fig. 3 shows the light baryon (left) and the charmonium (right) spectrum for the subset of $J^{P C}$ in which candidate hybrids were identified in Refs. [7, 15]. A similar pattern of states and energy scale above the lightest conventional state in the spectrum is observed in light, heavy-light and heavy mesons and baryons and is discussed in more detail in Ref. [16]. The calculations shown here are carried out at a relatively heavy pion mass, $M_{\pi} \sim 400 \mathrm{MeV}$ and at fixed lattice spacing. Nevertheless, the common features found for a range of quark mass and for both mesons and baryons are striking.

\subsection{Scattering and resonance states}

Most hadrons are resonances, located close to or above strong decay thresholds and in a lattice calculation the relevant scattering parameters must be extracted indirectly. The most widely used approach 

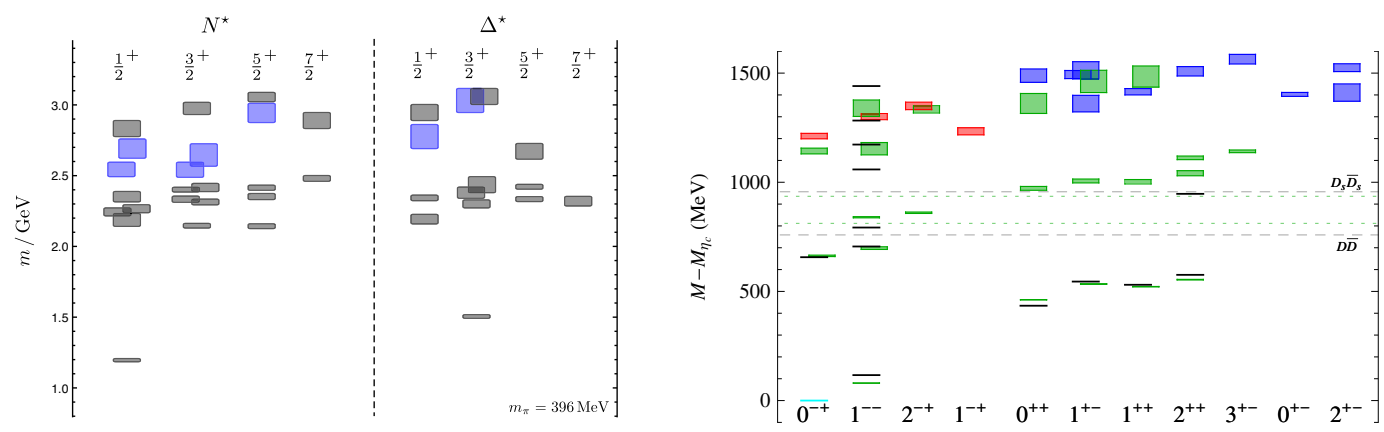

Figure 3. The left pane shows states identified as hybrids, colored blue, in the light baryon spectrum. The right pane shows charmonium hybrids, colored red and blue. The red states are identified as a hybrid supermultiplet, as described in the text, and the blue as hybrids that fall into an excited supermultiplet structure.

relates energies determined in a finite volume (the lattice simulation) to the infinite-volume scattering matrix amplitudes. The formalism, by Lüscher for $\pi \pi$ scattering in the elastic regime [17] has been generalised for moving frames, non-identical particles, multiple two-particle channels and particles with spin - see e.g. Ref. [18] and references therein. An alternative approach, by HAL QCD relies on potentials, determined in a lattice calculation, to extract the required scattering parameters - see Ref. [19] and references therein. While the Lüscher approach is used by a number of groups and has been extended and extensively tested including a recent coupled-channel calculation in the open-charm sector [20], the potential method remains at any earlier stage and is not, to date, widely used.

\subsubsection{A first look - X(3872)}

Early lattice investigations of the $X(3872)$ are described in Refs. [21-23] and summarised in Figure 4. These studies considered $D \bar{D}^{*}$ scattering using the Lüscher approach and find a pole just below threshold, in agreement with the experimental picture. A combination of single-meson and multi-meson operators were included and further study of the operator dependence [22] found that the threshold state (interpreted as the $X(3872)$ ) is not found if the single meson operators, corresponding to the $\chi_{c 1}(1 P)$, are omitted, implying that $c \bar{c}$ components are more important in the basis than the diquark-antidiquark components. It is important to note that these are exploratory calculations which should be improved before any definitive statements can be made.

\subsubsection{A first look $-Z_{c}^{+}(3900)$}

The charged state $Z_{c}^{+}$is manifestly exotic and does not fit in quark models. The precise nature of the state is as yet undetermined and it is hoped that lattice QCD can help to resolve this puzzle. However, a number of challenges must be faced: the $Z_{c}^{+}$lies above several thresholds and so decays to several two-meson final states. In principle this requires a coupled-channel analysis and in a lattice calculation the number of relevant coupled-channels is large for high energies. The state has been studied using the Lüscher approach by a number of groups [23-25] who find no $Z_{c}^{+}$candidate, below $4.2 \mathrm{GeV}$ in their analyses. It should be noted that these do not include coupled channel analyses and the dependence on the operator bases used has not been explored. More recently, the HAL QCD collaboration have 

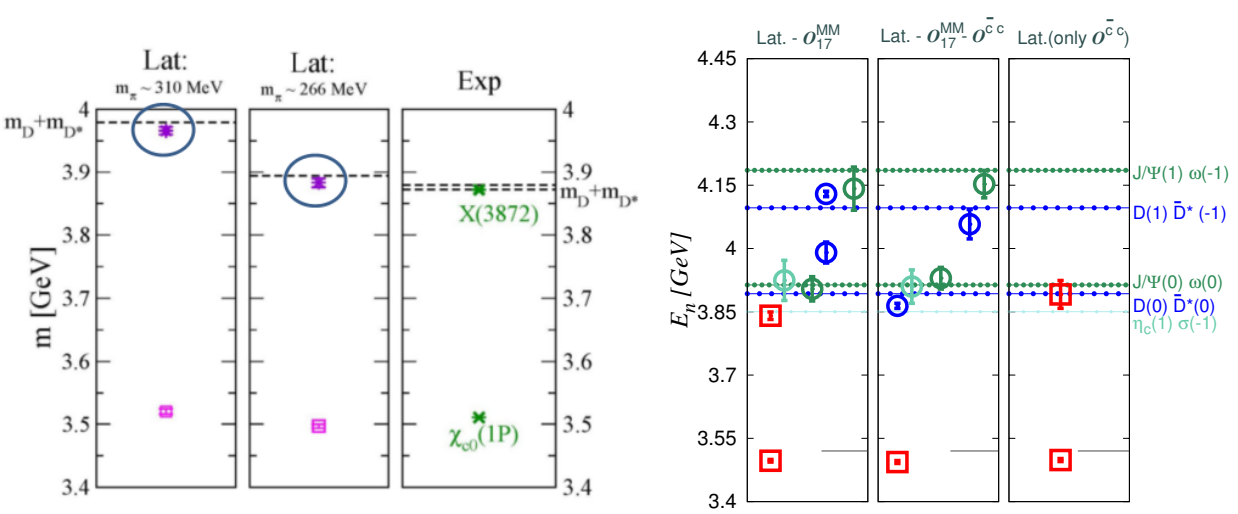

Figure 4. From Ref. [22]. The left pane shows the lattice determination of a state close to $D D^{*}$ threshold and the right pane illustrates the dependence on the operator basis: the state is missing when $c \bar{c}$ operators are removed.

studied this state in a coupled-channel analysis in their potential approach [26] and suggest the $Z_{c}^{+}$ appears as a threshold cusp, albeit for relatively heavy pions in their simulation. It is still too early for a definitive statement from lattice and more work is certainly motivated by these results.

\section{Summary and outlook}

I have highlighted the recent progress in lattice hadron spectroscopy. Precision calculations of the low-lying mesons and baryons, with all systematic uncertainties under control are now available. However, the most dramatic progress has been in calculations of excited and exotic states and the treatment of resonances and scattering. As methods are developed and refined sophisticated determinations of resonance properties can be expected - and are already available in some cases, as discussed here. While the numerical precision in current calculations is already very impressive controlling the "traditional" systematic uncertainties remains a challenge - as an example, scattering calculations with physical pion masses presents a challenge as the lighter quark masses open more decay channels which should be included. In charmonium, a proliferation of thresholds and coupled-channels complicates the lattice calculations and the inclusion of disconnected contributions remains a technical difficulty in that system. Nevertheless, theoretical developments continue e.g. in the treatment of three-coupled channel systems and work is ongoing on a numerical implementation. I expect that lattice calculations will be able to contribute meaningful and precise information about the nature of exotic states in QCD.

\section{Acknowledgements}

I would like to thank the organisers for the invitation to speak at this conference. I am grateful to my colleagues in the Hadron Spectrum Collaboration and acknowledge support of Science Foundation Ireland [RFP-PHY-3201].

\section{References}

[1] B. Blossier, M. Della Morte, G. von Hippel, T. Mendes, R. Sommer, JHEP 04, 094 (2009)

[2] D. Wilson, C. Liu (2016), Invited plenary talks at Lattice 2016

[3] A.S. Kronfeld, in 100 Years of Subatomic Physics, edited by E.M. Henley, S.D. Ellis (2013) 
[4] S. Borsanyi et al., Science 347, 1452 (2015)

[5] M. Peardon et al. (Hadron Spectrum), Phys. Rev. D80, 054506 (2009)

[6] H.W. Lin et al. (Hadron Spectrum), Phys. Rev. D79, 034502 (2009)

[7] L. Liu et al., JHEP 07, 126 (2012)

[8] G. Moir, M. Peardon, S.M. Ryan, C.E. Thomas, L. Liu, JHEP 05, 021 (2013)

[9] J.J. Dudek, R.G. Edwards, M.J. Peardon, D.G. Richards, C.E. Thomas, Phys. Rev. Lett. 103, 262001 (2009)

[10] J.J. Dudek et al., Phys. Rev. D83, 111502 (2011)

[11] J.J. Dudek, R.G. Edwards, P. Guo, C.E. Thomas (Hadron Spectrum), Phys. Rev. D88, 094505 (2013)

[12] R.G. Edwards, N. Mathur, D.G. Richards, S.J. Wallace (Hadron Spectrum), Phys. Rev. D87, 054506 (2013)

[13] M. Padmanath, R.G. Edwards, N. Mathur, M. Peardon, Phys. Rev. D91, 094502 (2015)

[14] M. Padmanath, R.G. Edwards, N. Mathur, M. Peardon, Phys. Rev. D90, 074504 (2014)

[15] J.J. Dudek, R.G. Edwards, Phys. Rev. D85, 054016 (2012)

[16] J.J. Dudek, Phys. Rev. D84, 074023 (2011)

[17] M. Luscher, Nucl. Phys. B364, 237 (1991)

[18] R.A. Briceno, Phys. Rev. D89, 074507 (2014)

[19] S. Aoki, B. Charron, T. Doi, T. Hatsuda, T. Inoue, N. Ishii, Phys. Rev. D87, 034512 (2013)

[20] G. Moir, M. Peardon, S.M. Ryan, C.E. Thomas, D.J. Wilson, JHEP 10, 011 (2016)

[21] S. Prelovsek, L. Leskovec, Phys. Rev. Lett. 111, 192001 (2013)

[22] M. Padmanath, C.B. Lang, S. Prelovsek, Phys. Rev. D92, 034501 (2015)

[23] S.h. Lee, C. DeTar, H. Na, D. Mohler (Fermilab Lattice, MILC) (2014)

[24] S. Prelovsek, C.B. Lang, L. Leskovec, D. Mohler, Phys. Rev. D91, 014504 (2015)

[25] Y. Chen et al., Phys. Rev. D89, 094506 (2014)

[26] Y. Ikeda, S. Aoki, T. Doi, S. Gongyo, T. Hatsuda, T. Inoue, T. Iritani, N. Ishii, K. Murano, K. Sasaki (2016), 1602.03465 